\title{
Static kinematics for an antagonistically actuated robot based on a beam-mechanics-based model
}

\author{
Agostino Stilli, Efstathios Kolokotronis, Jan Fraś, Ahmad Ataka, \\ Kaspar Althoefer, Helge A Wurdemann, Member, IEEE
}

\begin{abstract}
Soft robotic structures might play a major role in the $4^{\text {th }}$ industrial revolution. Researchers have successfully demonstrated advantages of soft robotics over traditional robots made of rigid links and joints in several application areas including manufacturing, healthcare and surgical interventions. However, soft robots have limited ability to exert higher forces when it comes to interaction with the environment, hence, change their stiffness on demand over a wide range. One stiffness mechanism embodies tendon-driven and pneumatic air actuation in an antagonistic way achieving variable stiffness values. In this paper, we apply a beammechanics-based model to this type of soft stiffness controllable robot. This mathematical model takes into account the various stiffness levels of the soft robotic manipulator as well as interaction forces with the environment at the tip of the manipulator. The analytical model is implemented into a robotic actuation system made of motorised linear rails with load cells (obtaining applied forces to the tendons) and a pressure regulator. Here, we present and analyse the performance and limitations of our model.
\end{abstract}

\section{INTRODUCTION}

With the growing interest in the use of soft materials [1] for the creation of highly dexterous robots, soft material robotics has established itself as an important research topic within soft robotics. Some roboticists argue that soft robotic technologies will play a key role in the $4^{\text {th }}$ industrial revolution [2], for safe human-robot interaction in manufacturing [3]-[5], healthcare [6], and minimally invasive surgery (MIS) [7]. Numerous proposals for novel flexible robots, based on soft and hybrid materials, are continuously emerging [8].

Although recent advances in soft and soft material robotics are notable and holding considerable promise to achieve what was not possible with traditional rigid-link robots, one important drawback remains: despite their morphological capabilities, they have limited ability to exert higher forces on the environment when required, hence, change their stiffness on demand over a wide range. In the search for the right trade-off between desired compliance and exertable force, researchers explored numerous approaches to enable on-demand stiffness tuning of soft robots. According to the recent comparative study presented in [9], Variable Stiffness Systems (VSSs) for soft robots can

A. Stilli is with the Dept. of Computer Science, University College London, London WC1E 7JE, UK (email: a.stilli@ucl.ac.uk).

E. Kolokotronis and H.A. Wurdemann are with the Dept. of Mechanical Engineering, University College London, London WC1E 7JE, UK.

A. Ataka is with the Centre for Robotic Research, Dept. of Informatics, King's College London, Strand, London WC2R 2LS, UK

J. Fras and K. Althoefer is with the School of Engineering and Materials Science, Queen Mary University of London, London E1 4NS, UK. be divided in two main groups: (i) Active VSSs: these VSSs provide on-demand stiffening using an antagonistic approach, i.e., the creation of stiffness by means of equilibrium between two or more forces, at least one of which is an active force and (ii) Semi-Active VSSs: these VSSs provide on-demand stiffening relying on their capability of intrinsically tuning the rigidity of the robotic system in which they are embedded. In our previous work [10]-[12] we have proposed a novel design for an inflatable continuum manipulator for applications in MIS based on tendon and pneumatic actuation, forming an active VSS. Systems based on this design can be highly compacted when in their undeployed, folded state and can be significantly expanded in volume by injecting fluid and changing their stiffness by several orders. Despite the aforementioned benefits, inflatable robots that can change their stiffness are typically more complex to model and control in comparison with their rigid counterparts.

The well-known multiple constant curvature model describes the body pose of a continuum manipulator in geometrical terms by approximating its bending with a series of constant curvature sections, as shown in [13]. This model allows the discretization of the continuous body reducing the robot's infinite Degrees of Freedom's into a finite number. This model has been extensively investigated [14], [15]. Its combination with the Euler-Bernoulli beam theory that considers a beam's deflation to be a result of pure bending has found applications in continuum robots, e.g., for active cannulas in MIS [16] and silicone-based soft robotics [17]. External physical interaction with the environment has not yet been investigated and modelled. The Cosserat rod theory approach has been proposed for the control of soft robotic manipulators due to their intrinsic non-linearity introduced by their material and geometrical properties [18]. Cosserattype rod methods are based on the assumption of a rod base curve as a deformable directed curve with attached deformable or non-deformable vectors [19]. The theory has been applied to continuous and finally soft systems [20]. However, simplifying assumptions such as the EulerBernoulli hypothesis are required when aiming for successful implementation in embedded control [21]. Aiming at modelling, in particular, soft robotic structures, the Finite Element Method (FEM) is used to discretise a soft body into several elements of constant stiffness [22]. Despite the recent advancements in FEM solvers the fast computation of the soft-robot's compliance matrix remains a challenge. The implementation of a multi-rate scheme in which lower frequency loops are used to capture the body's deformation while high refresh rates are used for its actuating system, have shown great potential [23]. 


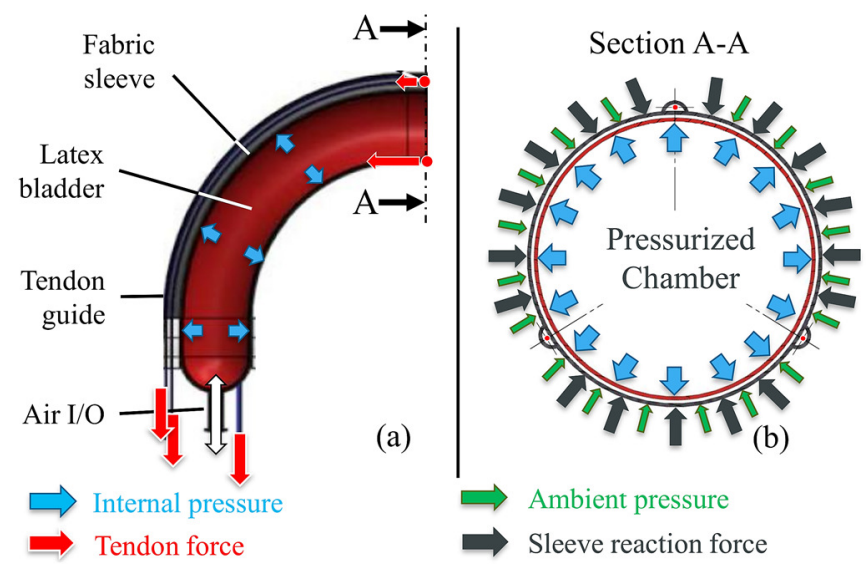

Figure 1 - CAD drawings of the soft, stiffness-controllable inflatable manipulator: (a) longitudinal section view showing the arm components and (b) cross-section view showing the tendon routing and their fixture points, the force distribution due to the air in pressure, the reaction force of the sleeve and the tendon pulling force.

These algorithms are computationally expensive for fast robot response if high deformation accuracy is required.

In this paper, a beam-mechanics-based model is validated for our inflatable soft, stiffness-controllable robotic manipulator firstly presented in [10]. The static mathematical model applies the Euler-Bernoulli beam model on discrete cross-sections/segments along the robot as described for a silicone-based, fluidically actuated manipulator in [17]. Applying this approach considers the wide range of stiffness values of the robot as well as forces exerted to the manipulator's tip in interaction with the environment. The kinematics are implemented and assessed for a soft robotic manipulator based on an active-active actuation technology: The inflatable manipulator uses tendon-driven and pneumatic air actuation in an antagonistic way to achieve a wide range of stiffness and elongation.

The paper is organised as follows: Section II recalls the soft, stiffness controllable robotic manipulator based on an antagonistic actuation principle. The Euler-Bernoulli beam model described in this section will be applied and validated using this robotic device. The estimation of the Young's modulus is obtained from experimental stiffness results. In Section III, the mechanical design of the overall robotic actuation system and implementation of the kinematic model is presented. To demonstrate the feasibility of the proposed beam-mechanics-based model, several experiments have been conducted (see Section IV). The results highlight the achievements of the proposed technique.

\section{Mathematical Model For a RoBot with AN ACtive VARIABLE STIFFNESS SYSTEM}

\section{A. Recalling the design of the inflatable robot}

The presented kinematic model has been adapted for the soft, stiffness-controllable robot based on an antagonistic actuation principle presented in [10]-[12]. Fig.1 shows the robotic manipulator longitudinal and cross-section view showing the tendon routing and their fixture points, the force distribution due to the air in pressure, the reaction force of the sleeve and the tendon pulling force.

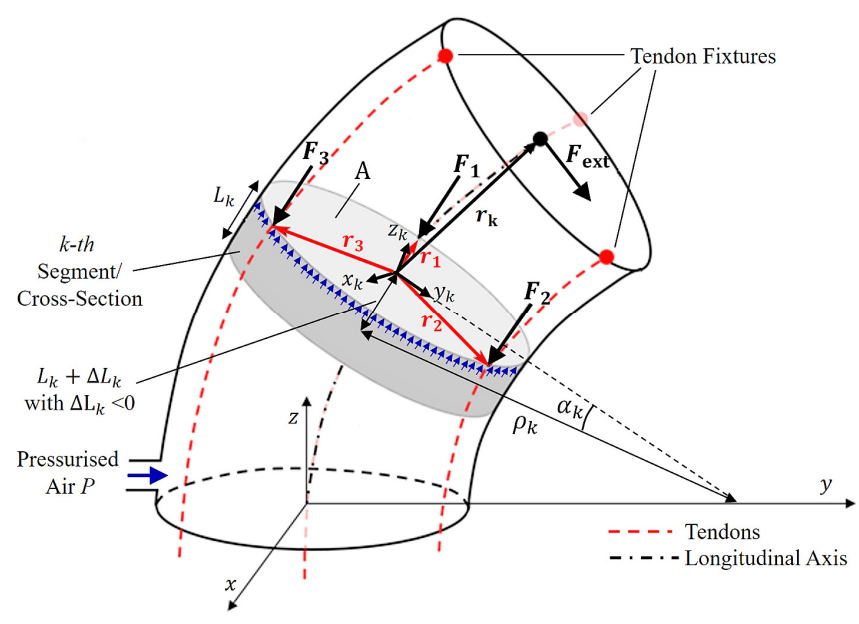

Figure 2 - The pressure and tendon forces exerted on an arbitrary manipulator's cross-section/segment. $P$ is the internal bladder's pressure $\boldsymbol{F}_{\mathbf{1}}, \boldsymbol{F}_{\mathbf{3}}$, and $\boldsymbol{F}_{\mathbf{3}}$ are forces applied by the tendons and $\boldsymbol{r}_{\mathbf{1}}, \boldsymbol{r}_{\mathbf{2}}$, and $\boldsymbol{r}_{\mathbf{3}}$ their position vector with respect to the centre of the cross-section. $\Delta L_{k}$ corresponds to the (negative) elongation resulting from the sum of forces.

The robot structure is composed of three main elements: an inner airtight and stretchable latex bladder, an outer, nonstretchable (but shrinkable) polyester fabric sleeve and three pairs of nylon tendons. The stretchable cylindrical latex bladder is inserted into the cylindrical polyester sleeve. The outer sleeve has a free length of $135 \mathrm{~mm}$ and has a diameter of $35 \mathrm{~mm}$, when fully inflated. As the fabric material is nonstretchable, the outer sleeve prevents any ballooning of the inner bladder in radial direction beyond the maximum diameter. Whilst morphing from a deflated state to an inflated state, the robot can only expand along its longitudinal axis (elongation). The stiffness of the arm can be controlled by adjusting the pressure, e.g., high air pressure results in stiffer and low pressure in softer states. The nylon tendons are guided along the outside of the manipulator sleeve within polyester channels, $120^{\circ}$ spaced apart along the perimeter of the outer sleeve and fixed to the tip of the manipulator.

\section{B. Kinematic model based on the Euler-Bernoulli beam model}

Soft robotic manipulators do not satisfy the constant curvature conditions [17]. When the manipulators are in physical interaction with the environment, the application of the constant curvature model results in large deviations between the theoretical model and experimental results. Hence, we present an Euler-Bernoulli beam model: the manipulator is discretised along the longitudinal axis into a number of segments. The Euler-Bernoulli beam theory is then applied individually to each segment before the manipulator's geometry is re-assembled. After the manipulator's discretization into $n$ cross-sections/segments, the first step is to calculate the elongation and bending moment of each individual segment when the tendons are actuated. When the manipulator is only pressurised, the pressurised air in the internal chamber keep the body pose straight. Each section in straight configuration has the same length $L_{k}$. Bending the manipulator by actuating one, two or three tendons leads to a contraction $\Delta L_{k}$ of the manipulator section in correspondence of its central axis (see Fig. 2). 
Using the Euler-Bernoulli equation:

$$
\kappa_{k}=\frac{1}{\rho_{k}}=\frac{\left|\boldsymbol{M}_{k}\right|}{E I_{k}}
$$

it is possible to obtain the section curvature $\kappa_{k} . \boldsymbol{M}_{k}$ corresponds to the acting moments on the section, $E$ to the material's Young modulus, $I_{k}$ to the beam's cross-section's second moment of area, and $\rho_{k}$ the radius of the $k$-th crosssection. The contraction caused by pulling the tendons and mathematically resulting from the net forces acting on the robot's cross-section can be approximated by Hooke's law in Equation (2).

$$
\Delta L_{k}=\frac{\left|\boldsymbol{F}_{T_{k}}\right|}{A E} L_{k}
$$

The net force vector $\boldsymbol{F}_{T_{k}}$ acts on the cross-section plane, $A$ (diameter $=35 \mathrm{~mm}, A=962.11 \mathrm{~mm}^{2}$ ) is the crosssection area, and $L_{k}$ the section's undeformed length. Assuming that the air pressure is homogenously distributed and tendons are moving without any friction inside the polyester channels, it becomes evident that the module of the net force vector $\boldsymbol{F}_{T_{k}}$ normal to the section surface for each arbitrary section can be obtained by the sum of the modules of the tendon force vectors $\boldsymbol{F}_{1}, \boldsymbol{F}_{2}$ and $\boldsymbol{F}_{3}$ on the one hand and the force by the pressurised air on the other hand. It yields:

$$
\left|\boldsymbol{F}_{T_{k}}\right|=P A-\left|\boldsymbol{F}_{1}\right|-\left|\boldsymbol{F}_{2}\right|-\left|\boldsymbol{F}_{3}\right|,
$$

where $P$ is the bladder's internal pressure. Similarly, the and the bending moment vector $\boldsymbol{M}_{k}$ can be obtained as:

$$
M_{k}=\boldsymbol{r}_{1} \times \boldsymbol{F}_{\mathbf{1}}+\boldsymbol{r}_{2} \times \boldsymbol{F}_{2}+\boldsymbol{r}_{3} \times \boldsymbol{F}_{3} .
$$

where $\boldsymbol{r}_{1}, \boldsymbol{r}_{2}$ and $\boldsymbol{r}_{3}$ are the position vectors shown in Fig. 2 . Given the two parameters for the resulting curvature and elongation, it is possible to describe the bending angle $\alpha_{k}$ for each section using Equation (5).

$$
\alpha_{k}=\int_{0}^{L_{k}+\Delta L_{k}} \frac{1}{\rho_{k}} d L_{k}
$$

If we consider only the forces applied by the tendons and the pressure, all the sections are subject to the same forces and momenta, thus they deform in the same way, hence they have the same curvature. In this case the system follows the constant curvature model. On the other hand, if we account for external forces that are acting at the manipulator's tip, Equations (3) and (4) must be extended to:

$$
\begin{gathered}
\left|\boldsymbol{F}_{T_{k}}\right|=P A-\left|\boldsymbol{F}_{1}\right|-\left|\boldsymbol{F}_{2}\right|-\left|\boldsymbol{F}_{3}\right|+\left|\boldsymbol{F}_{\text {ext, } z_{k}}\right| \\
\boldsymbol{M}_{k}=\boldsymbol{r}_{1} \times \boldsymbol{F}_{\mathbf{1}}+\boldsymbol{r}_{2} \times \boldsymbol{F}_{2}+\boldsymbol{r}_{3} \times \boldsymbol{F}_{3}+\boldsymbol{r}_{k} \times \boldsymbol{F}_{\text {ext }},
\end{gathered}
$$

where $\boldsymbol{F}_{\text {ext }}$ is the external force vector, $\boldsymbol{r}_{k}$ is the vector that connects the arbitrary cross-section's centre with the center of the manipulators' tip and $\boldsymbol{F}_{\text {ext, } z_{k}}$ is the component of $\boldsymbol{F}_{\text {ext }}$ in the $k$ cross-section's z-axis direction. In this case, $\boldsymbol{F}_{T_{k}}$ and $\boldsymbol{M}_{k}$ are different for each section, hence, each section has a different curvature $\kappa_{k}$ and bending angle $\alpha_{k}$.

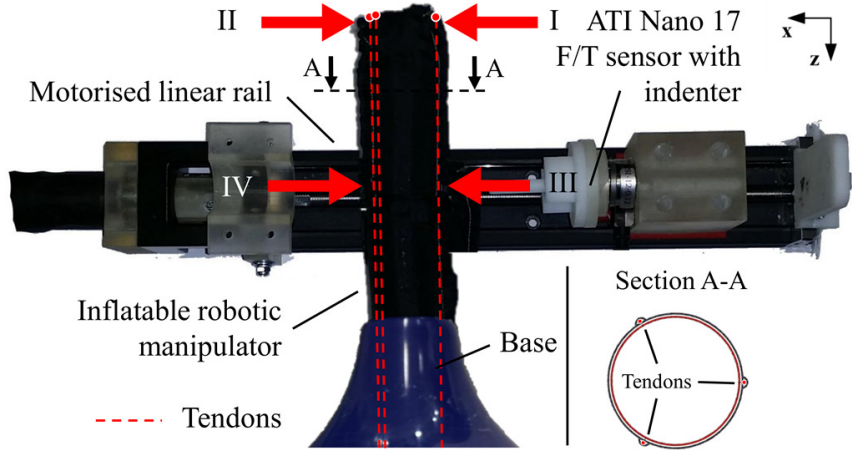

Figure 3 - Experimental setup using a motorised linear rail and an ATI Nano 17 Force/Torque sensor to measure force and displacement in order to determine the bending stiffness of the inflatable robotic manipulator when measured in correspondence of a tendon (I, III) and in between two adjacent tendons (II, IV), at the tip and in the middle.

\section{Experimental determination of the Young's modulus}

The presented analytical description of this soft, stiffnesscontrollable manipulator requires quantitative knowledge of its Young's modulus. This parameter of the presented beam-mechanics-based model varies depending on the pressure inside the robot's internal bladder. The determination of the Young's modulus is a significant challenge since the manipulator's structural characteristics prohibit the use of any analytical technique. However, the manipulator's bending stiffness $S_{x}$ along $x$ direction (Fig. 3) can be obtained experimentally and can be used to estimate the Young's modulus of our system. Generally, the bending stiffness is defined as the body's resistance against displacement forces [24]. A set of experiments was set up as shown in Fig. 3 allowing us to displace the inflatable robot at discrete locations along the manipulator using an ATI Nano17 Force/Torque (F/T) sensor, contextually measuring interaction forces and displacements. Both sides of the manipulator have been tested (on the tendon and in between two adjacent tendons) to understand how the tendon location affect the local and overall stiffness of our system. Given the boundary conditions of our experiment (cantilever beam), we assume that the bending stiffness $S_{x}$ of our system measured at the base (rigid) being infinite. Hence, we can now consider the inverse of the bending stiffness, the bending compliance $C_{x}$, which will then be zero in the base. Using the forcedisplacement data collected in the tests presented in Fig. 3 for the two sides of the manipulator we can now plot $C_{x}$, in function of the distance from the base $d_{\text {base }}$. These data are presented in Fig. 4 for different pressure levels $(15 \mathrm{kPa}$ and $30 \mathrm{kPa}$ ). As shown in the graphs, the bending compliance $C_{x}$ for this soft, stiffness-controllable robotic design is a linear function of the distance from the base $\left(d_{\text {base }}\right)$ and, as expected, the slopes of the lines are inversely proportional to the pressure level applied. Hence, given the linear trend of the bending compliance $C_{x}$, we can express the bending stiffness $S_{x}$ with the hyperbolic function shown in (8):

$$
S_{x}\left(d_{\text {base }}, P\right)=\frac{1}{m(P) * d_{\text {base }}}
$$




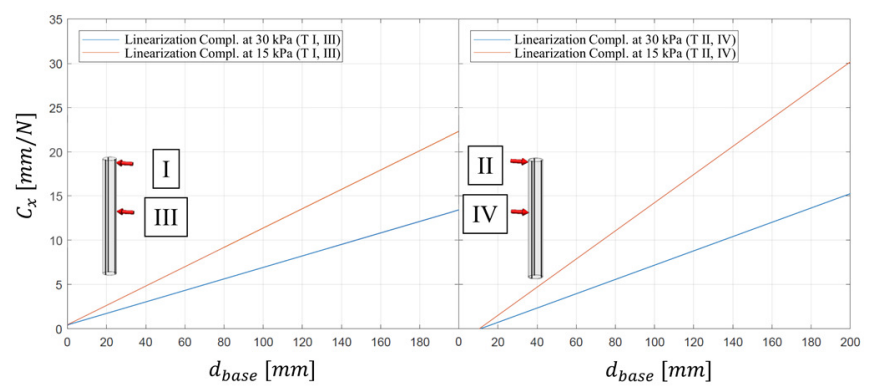

Figure 4 - Experimental determination of the manipulators' compliance (which is equivalent to the inverse of stiffness) as a function of the internal pressure and the distance from its base. The compliance values are measured and approximated for a straight configuration for tests I and III (left graph) and for tests II and IV (right graph) at $15 \mathrm{kPa}$ (orange) and 30 $\mathrm{kPa}$ (blue). For case I and III (left), the compliance is measured against one tendon and, for case II and IV (right), between two tendons.

Here $P$ is the internal bladder's pressure, $d_{\text {base }}$ is the distance from the manipulator's base to the location where the external force is applied and $m(P)$ is the angular coefficient of the lines (function of the pressure $P$ ) obtained by linearly approximating the compliance values obtained experimentally in the tests presented in Fig. 3 as shown in the graphs in Fig. 4. We can now use the measured bending stiffness $S_{x}\left(d_{\text {base }}, P\right)$ to find the Young's modulus of our system. For small deformations, we assume that our system behaves like a traditional cantilever beam. From classical mechanics, Equation (9) describes the relationship between the bending stiffness $S_{x}$ of a cantilever beam laterally loaded in function of the distance from the base $d_{\text {base }}$ where $S_{x}$ is measured, the Young's modulus $E$ and $I$ the second moment of area of (assuming the section circular $I=(\pi / 2) * r^{4}$ ).

$$
S_{x}\left(d_{\text {base }}\right)=\frac{3 E I}{d_{\text {base }}{ }^{3}}
$$

If we compare Equations (8) and (9), we can derive an equivalent Young's modulus for our inflatable robot $\left(E_{I R}\right)$ for our model.

$$
E_{I R}=\frac{d_{\text {base }}{ }^{2}}{3 \operatorname{Im}(P)}
$$

The equivalent Young's modulus $E_{I R}$ presented in Equation (10), differently from the traditional formulation of the Young's Modulus, is a function of the pressure $P$ inside the manipualtor, of the side on which we are interacting with the manipulator (accounting for the tendon position in respect to external force applied) and of the distance from the base.

\section{IMPLEMENTATION INTO A ROBOTIC ACTUATION SYSTEM}

Fig. 5 shows the setup of the robotic actuation system for validating the Euler-Bernoulli beam model described in Section II. The frame is made of three aluminium plates composing three of the six lateral faces of a hexagonal prism of $500 \mathrm{~mm}$ length. The inflatable robotic manipulator is mounted to the faceplate of the aluminium platform using a customised 3D-printed support made of Polylactic Acid (PLA) using an Ultimaker 2. A motorised linear actuator by C-Beam ${ }^{\mathrm{TM}}$ with an ACME Lead screw system is mounted to the inside of each aluminium plate. The linear rails have a length on $250 \mathrm{~mm}$.

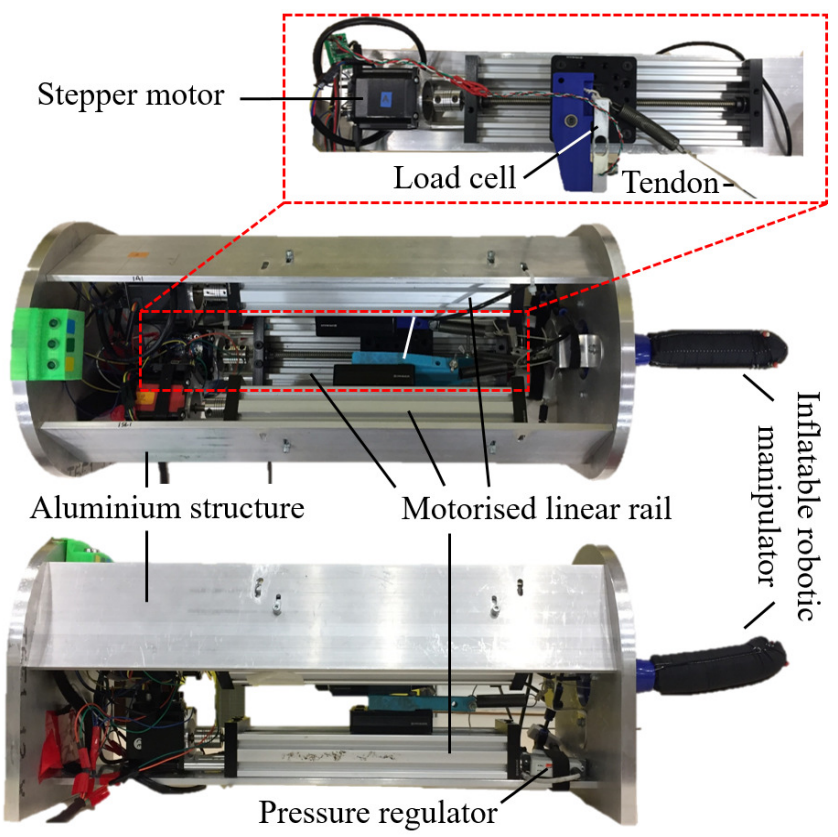

Figure 5: From bottom to top: Side and top view of the robotic actuation system for the soft, stiffness-controllable inflatable manipulator: A load cell is mounted on three motorised linear actuators by C-Beam ${ }^{\mathrm{TM}}$ (top). Each tendon of the robotic manipulator is connected to one load cell. The air inside the internal bladder is regulated using proportional pressure regulator.

Each actuator is driven by a $175 \mathrm{oz} 2.0$ A NEMA23 stepper motor with an on-board Arduino IDE. A load cell (0-50 N range) is mounted on the gantry plate of the linear actuators. A HX711 Weighing Sensors AD Module connects the force sensors to the available Arduino boards. The tendons of the manipulator are fed through the faceplate parallel to the $\mathrm{C}$ Beam $^{\mathrm{TM}}$ actuators and attached to each load cell. The inner bladder of the robot is connected to a $4 \mathrm{~mm}$ PVC pipe to an electronic proportional micro regulator (Camozzi K8P-SE522-2F). The regulator is able to control the air pressure up to $0.3 \mathrm{MPa}$, capable of inflating and deflating the inner bladder via one Arduino IDE. An air compressor (AS-186 Mini Piston Type) ensures the supply with sufficient pressure limited to the maximum pressure the regulators can cope with. The developer boards are connected via USB to a PC running the Robot Operating System (ROS). The MATLAB ROS Bridge allows to acquire force and pressure data as well as actuate the motorised linear actuator and pressure regulator.

\section{EXPERIMENTAL SETUP AND RESULTS}

\section{A. Experimental setup and test protocol}

Our beam-mechanics-based model for an inflatable soft, stiffness-controllable robot is validated by two sets of experimental tests: experiment 1 - the robotic manipulator's bending angle $\alpha$ is measured when forces are applied to one or two actuated tendons; experiment 2 - an external force $\boldsymbol{F}_{\text {ext }}$ is applied at the robot's tip, when in a straight configuration, and the deflection is recorded. As the stiffness values are experimentally determined, the internal pressure here follows the range between the discrete values of $15 \mathrm{kPa}$ and $30 \mathrm{kPa}$ and maximum displacements at the tip of $15 \mathrm{~mm}$. 


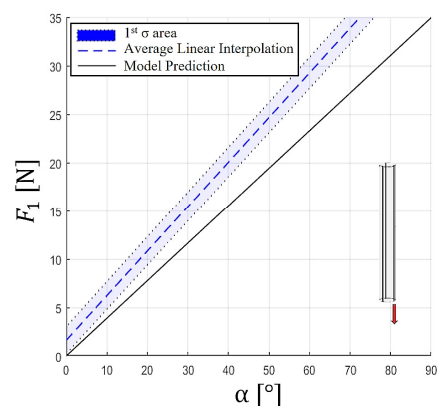

(a)

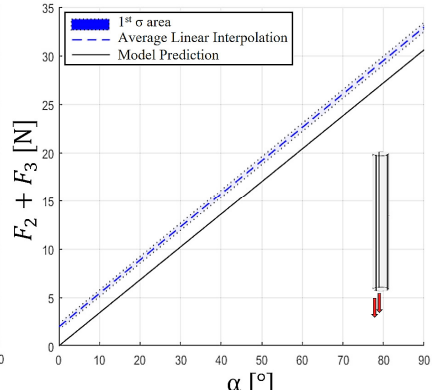

(b)
Figure 6: Experimental results compared to the beam-mechanics-based model: Bending angle versus forces applied to one (left) and two (right) tendons at $15 \mathrm{kPa}$. (a)

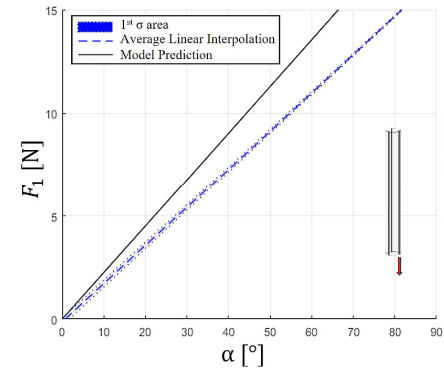

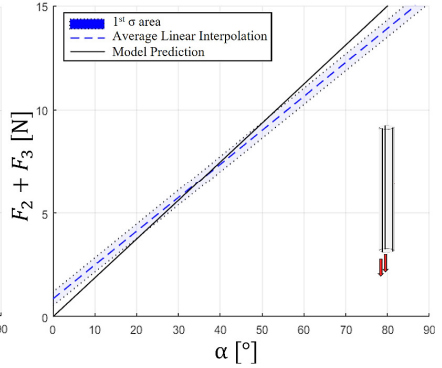

(b)
Figure 7: Experimental results compared to the beam-mechanics-based model: Bending angle versus forces applied to one (left) and two (right) tendons at $30 \mathrm{kPa}$.

Due to the symmetry of the inflatable manipulator, singleand dual-tendon actuation was analysed. The robotic actuation system was set up so that the bending and deflection motion moved within a planar surface. A Nikon D3300 DSLR camera was arranged with its image plane parallel to the aforementioned surface. A continuous series of images were recorded of five iterations for each experiment, converted to binary images based on threshold adjustments and analysed using the Image Processing Toolbox by MATLAB. Initial investigation of the trade-off between computational effort versus model accuracy resulted in the choice of $n=4$ cross-sections/segments for the analytical model (see Section IV-B and C) which then compared to experimental tests. This value has been experimentally chosen as the optimal one for the tendon forces, bending angles and manipulator displacement investigated.

\section{B. Experiment 1: Bending angle versus force applied to tendons}

The results of Experiment 1 continuously measuring the bending angle and force applied to one and two tendons are shown in Fig. 6 and 7. The results of the analytical beammechanics-based model are displayed by black linear curves. A linear approximation has been applied to the experimental raw data represented by blue curves including the shaded area along these curves showing the standard deviation. Fig. 6 and 7 illustrate the comparison of the results for $15 \mathrm{kPa}$ and $30 \mathrm{kPa}$ respectively. The theoretical model determines a displacement of $66^{\circ}$ and $80^{\circ}$ when applying $15 \mathrm{~N}$ to one in Fig. 6 (a) and two tendons in Fig. 6 (b), respectively. Experiments result in $82^{\circ}$ and $85^{\circ}$, with a slightly higher standard deviation when two tendons are actuated together.

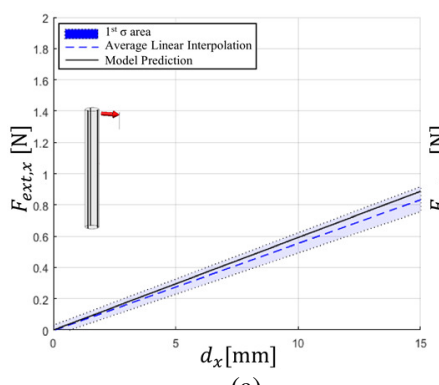

(a)

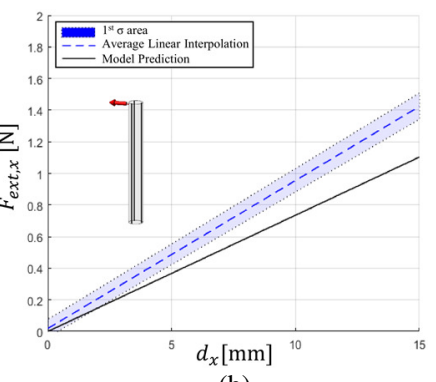

(b)
Figure 8: Experimental results compared to the beam-mechanics-based model: Displacement versus forces applied to one (left) and two (right) tendons of the manipulator's tip at $15 \mathrm{kPa}$.

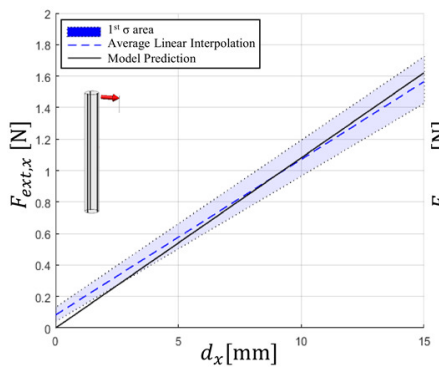

(a)

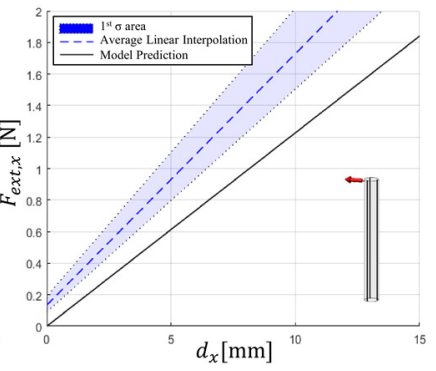

(b)
Figure 9: Experimental results compared to the beam-mechanics-based model: Displacement versus forces applied to one (left) and two (right) tendons of the manipulator's tip at $30 \mathrm{kPa}$.

For a pressure of $30 \mathrm{kPa}$ in Fig. 7, a force of $15 \mathrm{~N}$ results in an analytical bending angle of $39^{\circ}$ and $45^{\circ}$. A force of $30 \mathrm{~N}$ achieves an angle of $77^{\circ}$ and $88^{\circ}$, respectively. The experimental results return angles of $30^{\circ}$ and $38^{\circ}$ for $15 \mathrm{~N}$ and $62^{\circ}$ and $81^{\circ}$ for $30 \mathrm{~N}$ force applied to the tendons. For singletendon actuation in Fig. 6 (a) and Fig. 7 (a), the mathematical model generally returns larger bending angles for $15 \mathrm{kPa}$ and smaller bending angles for $30 \mathrm{kPa}$ compared to the experimental results. The error between the predicted and actual angles increase with larger bending angles. On the other side, values for the bending angle of the analytical model and experimental data set for two-tendons actuation (Fig. 6 (b) and Fig. 7 (b)) are in alignment. In fact, there is an offset of $7^{\circ}$ between the experimental results and the beammechanics-based model in Fig. 7 (b).

\section{Experiment 2: Displacement versus force applied to the manipulator's tip}

In Fig. 8 and Fig. 9, the results of Experiment 2 are shown: The manipulator is configured in a straight position and external forces are applied to the robot's tip achieving maximum displacements of $15 \mathrm{~mm}$. Instead of exerting pushing forces as shown in Fig. 3, the manipulator's tip is pulled towards one tendon in Fig. 8 (a) and Fig. 9 (a) and towards two tendons in Fig. 8 (b) and Fig. 9 (b) during Experiment 2. At $15 \mathrm{kPa}$ (see Fig. 8), the theoretical beam model returns displacements of $15 \mathrm{~mm}$ when a force of $0.9 \mathrm{~N}$ and $1.1 \mathrm{~N}$ is applied to one and two tendons, respectively. The linear approximation gives force values of $0.85 \mathrm{~N}$ and $1.45 \mathrm{~N}$ for these cases. Fig. 9 illustrates the results for a pressure of $30 \mathrm{kPa}$. Our implemented model estimated a force of $1.6 \mathrm{~N}$ and $1.85 \mathrm{~N}$ for a displacement of $15 \mathrm{~mm}$. These 
values are $1.55 \mathrm{~N}$ and $2.5 \mathrm{~N}$ looking at the approximations of the experimental data.

As pulling forces are applied to the manipulator's tip, the stiffness of the inflatable robot is provided by the opposing tendon(s). For instance, when external forces pull the manipulator's tip towards a single tendon as shown in Fig. 8 (a), the two opposite tendons counterbalance this physical interaction. Experimental data for two tendons providing stiffness counterbalance closely matches the results by our developed mathematical model (Fig. 8 (a) and Fig. 9 (a)). According to the results in Fig. 8 (b) and Fig. 9 (b), stiffness by one tendon only returns larger standard deviations and discrepancies between the theoretical and experimental data increase with larger displacements.

\section{Discussion}

It can be concluded that our model is in alignment with the experimental results for small bending angles and displacements. Non-linear effects might increase with larger bending angles and displacements which cannot be captured with this beam-mechanics-based model. Another limitation results from the experimental determination of the Young's modulus. The stiffness of the inflatable manipulator covers a wide range of values - in particular, when the manipulator is arranged in different configurations. The choice of the number of cross-sections in the model plays a key role in the reliability of this approach to predict and control the manipulator behaviour.

\section{CONCLUSIONS}

Building on our previous work, we have now adapted a beam-mechanics-based model for our soft, stiffnesscontrollable robotic manipulator. This mathematical model applies the Euler-Bernoulli beam model on discrete crosssections/segments along the robot. A robotic actuation system is set up measuring the force applied to the tendons of our inflatable robot and the pressure inside the internal bladder. Two types of experiments have been conducted to validate the proposed model: In Experiment 1, we evaluated the case of active forces applied to the manipulator by pulling the tendons, measuring the bending angle of the manipulator axis; in Experiment 2, external forces were applied at the tip of the manipulator measuring its displacement along the direction for application of the force. The Young's modulus of this analytical model is obtained from experimental data as the stiffness of the manipulator changes over a wide range.

In future work, the sensitivity of the mathematical model with respect to the choice of the number of cross-sections in the model will be explored. A parametrised model accounting for pressure levels and bending angles might be worth investigating, along with exploring an analytical model to calculate the robot's Young's modulus. Friction between the tendons and fabric material as well as arbitrary folding patterns of the fabric material might lead to high inaccuracies for large bending angles. These effects should be considered when further developing the analytical model.

\section{REFERENCES}

[1] S. Bauer, S. Bauer-Gogonea, I. Graz, M. Kaltenbrunner, C. Keplinger, and R. Schwödiauer, "25th anniversary article: a soft future: from robots and sensor skin to energy harvesters," Adv. Mater., vol. 26, no. 1, pp. 149-162, 2014.

[2] J. Rossiter and H. Hauser, "Soft robotics-the next industrial revolution," IEEE Robot. Autom. Mag, vol. 23, pp. 17-20, 2016.

[3] A. Stilli, H. A. Wurdemann, and K. Althoefer, "A Novel Concept for Safe , Stiffness-Controllable Robot Links," Soft Robot., p. soro.2016.0015, 2016.

[4] A. Stilli, L. Grattarola, H. Feldmann, H. A. Wurdemann, and K. Althoefer, "Variable Stiffness Link (VSL): Toward inherently safe robotic manipulators," in Robotics and Automation (ICRA), 2017 IEEE International Conference on, 2017, pp. 4971-4976.

[5] R. Pfeifer, H. G. Marques, and F. Iida, "Soft robotics: the next generation of intelligent machines," in Proceedings of the Twenty-Third international joint conference on Artificial Intelligence, 2013, pp. 5-11.

[6] M. A. Horvath et al., "An intracardiac soft robotic device for augmentation of blood ejection from the failing right ventricle," Ann. Biomed. Eng., vol. 45, no. 9, pp. 2222-2233, 2017.

[7] A. Arezzo et al., "Total mesorectal excision using a soft and flexible robotic arm: a feasibility study in cadaver models," Surg. Endosc., vol. 31, no. 1, pp. 264-273, 2017.

[8] H. Lipson, "Challenges and opportunities for design, simulation, and fabrication of soft robots," Soft Robot., vol. 1, no. 1, pp. 21-27, 2014.

[9] M. Manti, V. Cacucciolo, and M. Cianchetti, "Stiffening in soft robotics: A review of the state of the art," IEEE Robot. Autom. Mag., vol. 23, no. 3, pp. 93-106, 2016.

[10]A. Stilli, H. A. Wurdemann, and K. Althoefer, "Shrinkable, stiffnesscontrollable soft manipulator based on a bio-inspired antagonistic actuation principle," in IEEE International Conference on Intelligent Robots and Systems, 2014, pp. 2476-2481.

[11]A. Stilli, F. Maghooa, H. Wurdemann, and K. Althoefer, "A new bioinspired, antagonistically actuated and stiffness controllable manipulator," in Workshop on Computer/Robot Assisted Surgery (CRAS), 2014.

[12]H. A. Wurdemann, A. Stilli, and K. Althoefer, "Lecture notes in computer science: An antagonistic actuation technique for simultaneous stiffness and position control," in International Conference on Intelligent Robotics and Applications, 2015, pp. 164-174.

[13]M. W. Hannan and I. D. Walker, "Kinematics and the implementation of an elephant's trunk manipulator and other continuum style robots," J. Robot. Syst., vol. 20, no. 2, pp. 45-63, 2003.

[14] I. S. Godage, G. A. Medrano-Cerda, D. T. Branson, E. Guglielmino, and D. G. Caldwell, "Dynamics for variable length multisection continuum arms," Int. J. Rob. Res., vol. 35, no. 6, pp. 695-722, 2016.

[15] V. Falkenhahn, T. Mahl, A. Hildebrandt, R. Neumann, and O. Sawodny, "Dynamic modeling of bellows-actuated continuum robots using the Euler-Lagrange formalism," IEEE Trans. Robot., vol. 31, no. 6, pp. 1483-1496, 2015.

[16]R. J. Webster III, J. M. Romano, and N. J. Cowan, "Mechanics of precurved-tube continuum robots," IEEE Trans. Robot., vol. 25, no. 1, pp. 67-78, 2009.

[17] J. Fraś, J. Czarnowski, M. Ma, and J. Główka, "Static modeling of multisection soft continuum manipulator for Stiff-Flop project," pp. 113.

[18]M. B. Rubin, Cosserat theories: shells, rods and points, vol. 79. Springer Science \& Business Media, 2013.

[19]E. W. Hawkes, L. H. Blumenschein, J. D. Greer, and A. M. Okamura, "A soft robot that navigates its environment through growth," Sci. Robot., vol. 2, no. 8, Jul. 2017.

[20]F. Renda, M. Giorelli, M. Calisti, M. Cianchetti, and C. Laschi, "Dynamic model of a multibending soft robot arm driven by cables," IEEE Trans. Robot., vol. 30, no. 5, pp. 1109-1122, 2014.

[21]F. Renda, M. Cianchetti, M. Giorelli, a Arienti, and C. Laschi, "A 3D steady-state model of a tendon-driven continuum soft manipulator inspired by the octopus arm.," Bioinspir. Biomim., vol. 7, no. 2, p. 025006 , Jun. 2012.

[22]C. Duriez, "Control of elastic soft robots based on real-time finite element method," in Robotics and Automation (ICRA), 2013 IEEE International Conference on, 2013, pp. 3982-3987.

[23]F. Largilliere, V. Verona, E. Coevoet, M. Sanz-Lopez, J. Dequidt, and C. Duriez, "Real-time control of soft-robots using asynchronous finite element modeling," in ICRA 2015, 2015, p. 6.

[24]J. S. Przemieniecki, Theory of matrix structural analysis. Courier Corporation, 1985. 